\title{
Olfactory coordinated area coverage
}

\author{
Svetlana Larionova $\cdot$ Nuno Almeida $\cdot$ Lino Marques • \\ A. T. de Almeida
}

Published online: 8 June 2006

(C) Springer Science + Business Media, LLC 2006

\begin{abstract}
This paper proposes an olfaction based methodology to automatically cover an unknown area enabling the decoupled cooperation of a group of floor cleaning mobile robots. This method is based on the utilisation of low cost chemical sensors in cleaning mobile robots, in order to differentiate clean from dirty areas. The experimental results show that the use of olfactory capabilities allows to efficiently cover and clean a certain area, and demonstrate the possibility of coordinating several mobile robots without the need of expensive sensing capabilities, map building or complex algorithms for task scheduling.
\end{abstract}

Keywords Complete coverage - Robot olfaction . Cleaning robots $\cdot$ Robot cooperation

\section{Introduction}

Covering large areas is a task that is obviously useful in a large number of applications such as cleaning large surfaces (e.g. supermarkets, airports, shopping centres, etc.), determining the spatial distribution of a parameter such as metal concentration in the ground or explosive vapours concen-

S. Larionova $(\bowtie) \cdot N$. Almeida $\cdot$ L. Marques $\cdot$ A. T. de Almeida Institute of Systems and Robotics, Department of Electrical and Computers Engineering, University of Coimbra,

Portugal

e-mail: sveta@isr.uc.pt

N. Almeida

e-mail: nuno@isr.uc.pt

L. Marques

e-mail: lino@isr.uc.pt

A. T. de Almeida

e-mail: adealmeida@isr.uc.pt tration at a near ground level in demining applications, or simply for mapping purposes (Choset, 2001). The efficiency of these tasks can be largely increased if they are performed by multiple robots instead of a single one. Enabling cooperation through the use of chemical information is a common procedure in several animal societies such as ants or bees. In this paper it is proposed an algorithm to enable decoupled cooperative coverage of a certain area by multiple robots based on the use of chemical information, keeping in mind that for both cleaning and demining it is essential to guarantee total coverage of the area of interest.

Employing more than one robot decreases the coverage time, although this option increases the complexity of the overall control. Using chemical information will enable the implementation of a decoupled cooperation technique which will allow scalability without increasing the complexity of the system. If each robot can leave a chemical mark along its path and all robots are able to sense it, there is no need for map building or to implement a global control layer to coordinate the robots. This principle is easily implementable on floor cleaning robots using the fact that after cleaning, water and cleaning chemicals are released on the floor and might be used as a mark that can be detected by chemical sensors.

\subsection{Covering algorithms}

Several approaches are possible for covering an unknown environment with mobile robots. The most common solution is to implement cellular decomposition of the target area. In approximate cellular decomposition, the working area is divided into cells of equal size. Gabriely and Rimon (2002) proposed the following approach for online covering of an unknown environment: the working area is incrementally 
divided into disjoint cells organized in a spanning tree whose grid is sequentially followed by the robot. This algorithm provides a complete coverage path but the resulting path has a large number of turns which makes difficult its practical implementation. Another group of methods use exact cellular decomposition in which the work area is divided into cells with different sizes and shapes, in such a way that the total area of cells is equal to the initial area. The decomposition must be done guaranteeing that each resulting cell in this decomposition can be covered using back and forth motion. The advantage of this algorithm compared with the former is that it can be implemented with a more regular path and with a lower number of turns. Choset and Pignon (1997) proposed an algorithm to make the exact cellular decomposition of a free area using a virtual line (also called slicing line) that moves across the map, splitting it into smaller areas. Cells are formed each time the virtual line makes a tangent with the bound of an obstacle. Then each cell is covered using back and forth motions. For the online implementation of this algorithm it is important to find all points on the obstacle bounds where cells must be formed. Acar and Choset (2000) proposed two methods to sense these points using robot range sensors. Hert et al. (1996) proposed an algorithm which does not use cellular decomposition, but incrementally explores the area and covers it using back and forth motions. Here the environment is represented as a combination of inlets and islands that are investigated during the exploration.

Some previous work have already used chemical or ink marks left by robots to obtain information about areas already covered. Mann and Katz (1999) proposed an odour based coverage algorithm for cleaning robots using a trace following technique which assumes that the sensor can be rotated. Svennebring and Koenig (2003) implemented a coverage algorithm for trail-laying robots including obstacle-avoidance and trail-avoidance behaviours. In this work, the authors used coloured trails and optical sensors to detect the trails. The main difference between this and previous works is the employment of trail marks in an exact cellular decomposition technique. This method can guarantee complete coverage of the area, and allows the utilization of multiple robots without a large increase of multiple passages on the same area.

\section{Methodology}

The usage of olfaction marks can simplify coverage path planning and also cooperative coverage. This simplification is based on several aspects:

1. The first one is the assumption that each robot leaves marks along its path. It is reasonable to use odour marks especially in the case of cleaning robots because usually the water and the cleaning substance released on the floor by the robot can be sensed by chemical sensors. Thus, for a team of robots, the olfaction field is a source of information about the coverage performed by other robots.

2. The dispersion of the cleaning vapours creates an odour field that changes in space and time. This property is useful to let the vapours vanish along the time, but makes difficult the modelling and analysis of the measured field. It is therefore important that the chemical sensor setup provides a good spatial resolution of the chemical trails.

3. The exact cellular decomposition method is the base idea of the suggested coverage algorithm. As described before, each cell in this decomposition is covered by back and forth motions, so, the area covered is always bounded and filled i.e., if one robot senses the bound of a cleaned area, it can be sure that all the area inside the sensed bound was already cleaned.

Taking these assumptions into consideration, the following online coverage algorithm can be used by one or several robots.

\subsection{Area coverage algorithm}

The main task of the robot is to divide the surrounding free area into cells in such a way that each cell can be covered using simple back and forth motions (boustrophedon decomposition). As there is no map available, the decomposition should be incremental i.e., during the robot movements the surrounding conditions have to be analysed in order to recognise the situation in which the area should be decomposed. This approach does not need any previous knowledge about the working area or any additional exploration of it. Each time a new cell is obtained by decomposition it should be covered using back and forth motions. When all cells are covered and no new cell can be created, the coverage is completed.

\subsubsection{Decomposition principle}

The concept of slicing line is used to obtain the boustrophedon decomposition. This is a virtual line which is parallel to the straight motions of the boustrophedon path. The main principle is: if the slicing line discovers an obstacle which breaks it (bounding point in Fig. 1) then the area should be decomposed. When the robot moves straight forward along the slicing line, a bounding point can be identified by the range sensors as the local minimum of the distance to the nearest obstacle (see Fig. 4). While covering the current cell, the robot senses these points in both side directions and when the current cell is covered new cells are created using them. It should be noticed that the range information is not 


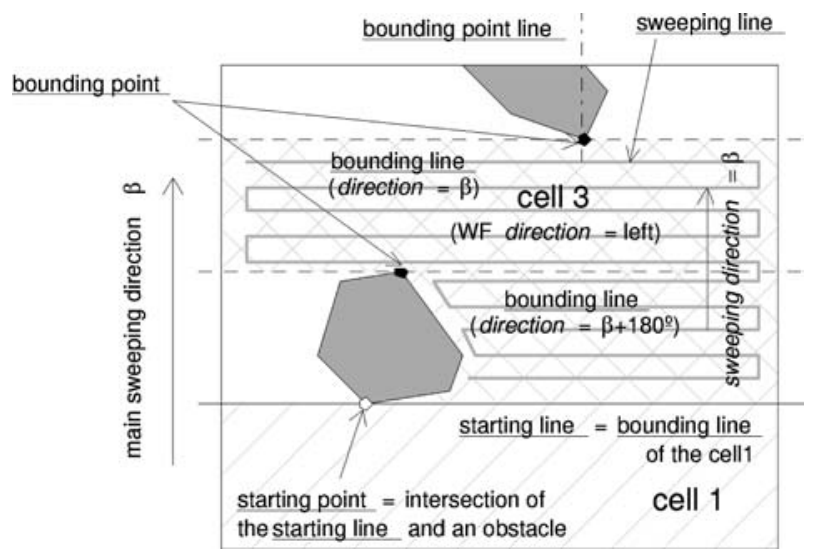

Fig. 1 Parameters of the cells and its bounding lines

considered to be precise. Thus the bounding point is used only as an approximate position in which the robot will start a wall-following path as it will be described later.

\subsubsection{The algorithm}

The important goal of this work is to develop an algorithm which can be implemented in real conditions and in unknown environments. It is assumed that the odometry of the robot and its range sensors are far from perfect. Thus the algorithm does not consider the creation of a map based only on the localization system. Instead, the important points and lines (Fig. 1) that compose a topological map are used only as approximate positions where the robot performs motions in relation to obstacles. In this case small odometry errors do not cause any problems for the algorithm performance. Obeying to this concept, each cell in the decomposition is defined as having the following elements (Fig. 1):

Starting point determines where the wall-following path should be started to guide the robot to the start of the cell

Starting line denotes the start of the cell. When it is intercepted while moving from the starting point, the coverage of the cell should be started

Bounding lines and bounding points determine the beginning of new cells

Wall-Following (WF) direction defines the side of the robot by which it should perform the wall-following path to start the cell

Sweeping direction can be equal to main sweeping direction or opposite to it.

The general structure of the algorithm, represented in Fig. 2, contains the decomposition of the area into cells and their coverage in the order they were created. The algorithm, explained in the diagrams of Fig. 2 to 5, is subdivided into the following tasks:

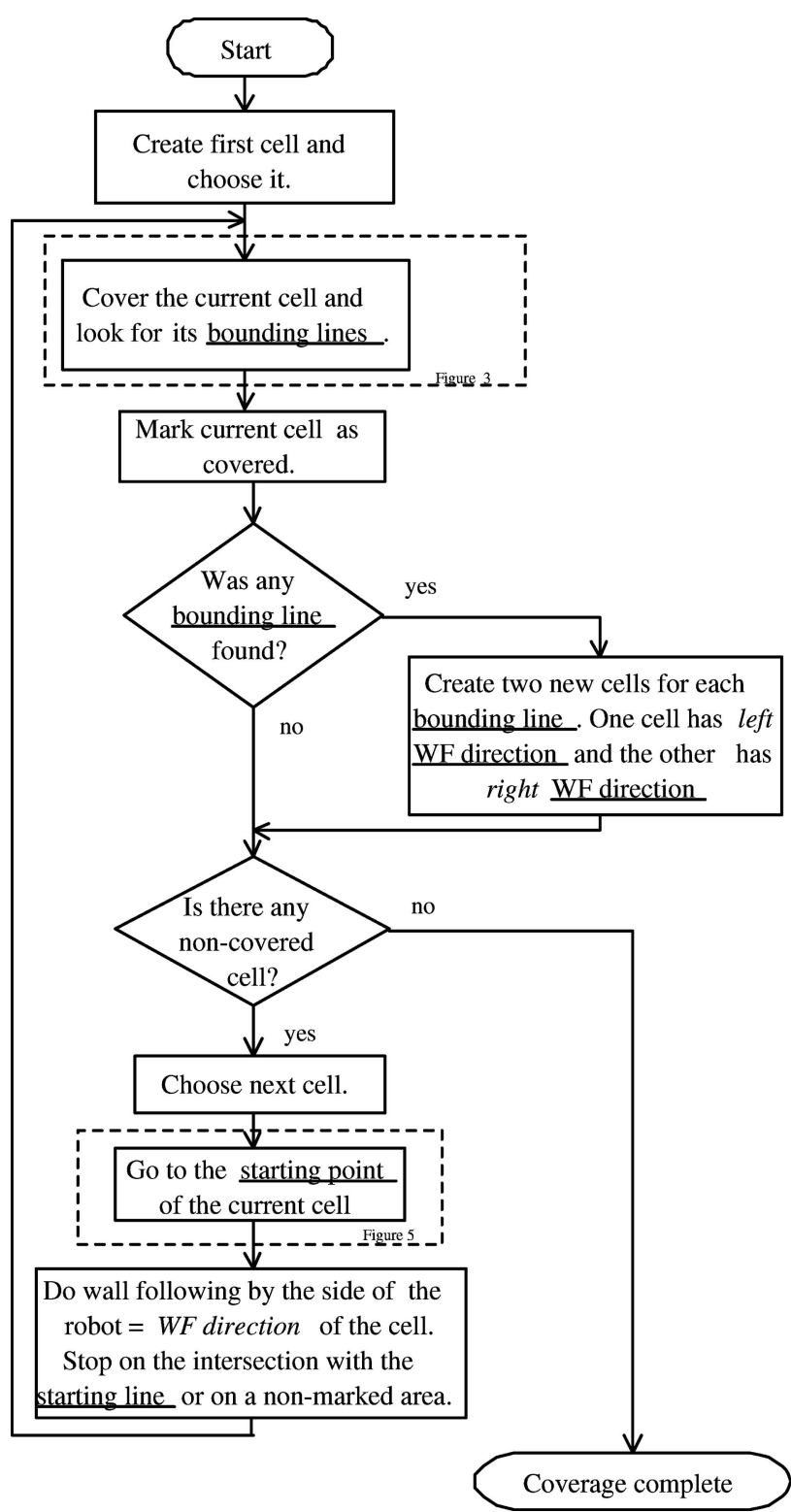

Fig. 2 Coverage algorithm

Starting a cell. After choosing the next non-covered cell in the list, the path to its starting point is computed by analysing the previously covered cells and their lines (see Fig. 5). When the robot is situated near the starting point of the cell, it performs a wall-following motion by the side of the robot equal to WF direction. When the starting line is intercepted, the cell coverage can be started.

Cell coverage. Each cell is covered using simple back and forth motions (Fig. 2). The main sweeping direction with angle $\beta$ (Fig. 1) can be defined or is automatically computed by $\beta=\alpha+90^{\circ}$ where $\alpha$ is the initial angle of the robot. For each cell the sweeping direction of 
Fig. 3 Cell coverage. $(*)$ Set direction for the straight-line motion: angle $=$ sweeping direction $\pm 90^{\circ}$ away from the nearest obstacle; Set behaviour to straight-line. $(* *)$ Make next sweeping line at a distance equal to the size of the robot towards the sweeping direction; set behaviour to wall-following

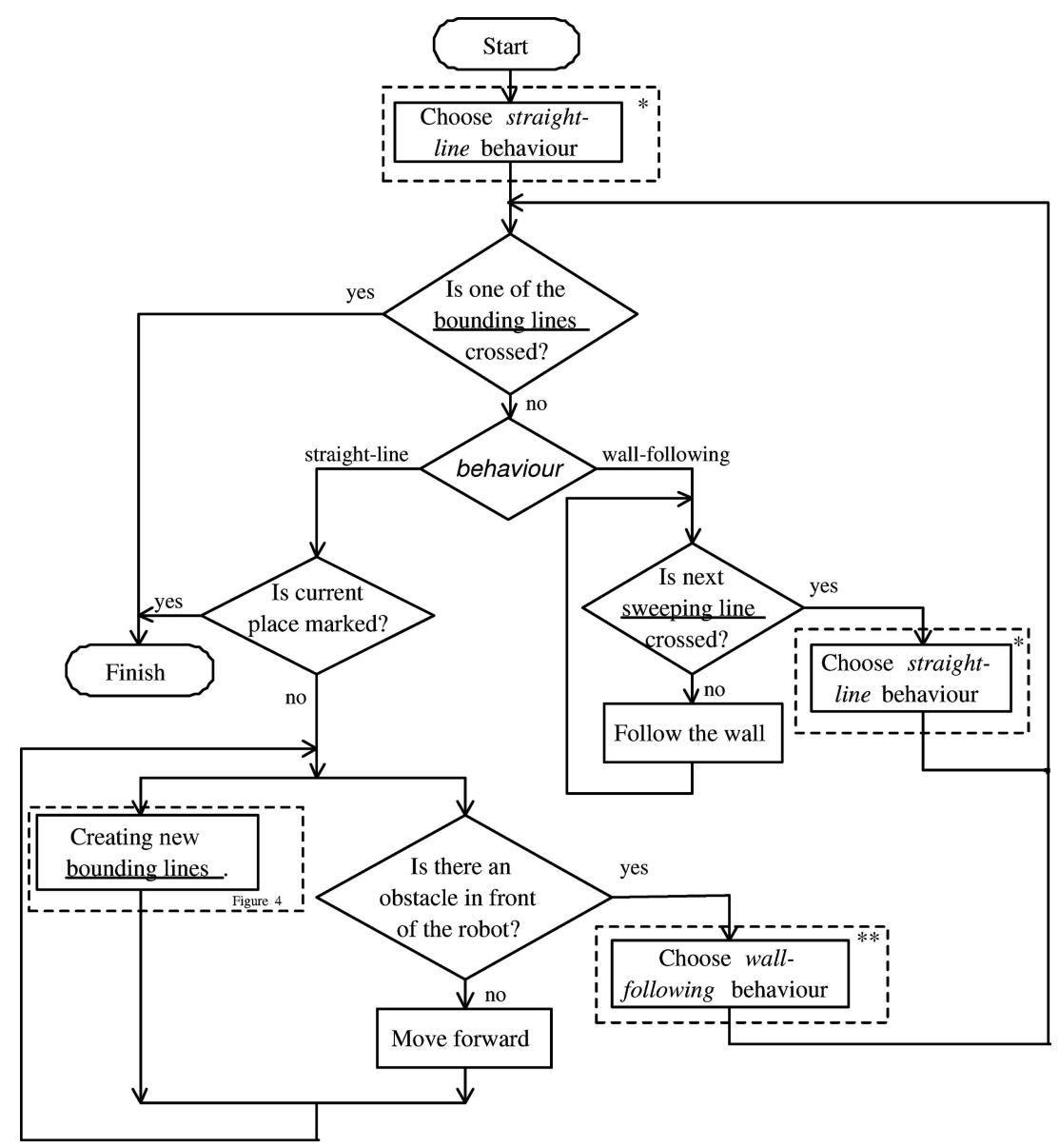

coverage can be $\beta$ or $\beta+180^{\circ}$. The direction of back and forth motions is then given by $\alpha$ or $\alpha+180^{\circ}$. The bounding lines which divide the area into cells are parallel to $\alpha$.

Bounding line check. While covering a cell, the robot checks its bounding lines using range sensors. When a cell is covered, each pair of bounding line and bounding point creates two new cells, becoming the starting line and the starting point for each respective new cell.

\subsubsection{Utilization of olfaction}

To recognise that a cell was covered, a robot has to verify different situations:

- One of the bounding lines is intercepted. This means that the area must be decomposed, thus new cells are created and the current cell is marked as covered.

- The cell or part of it has already been covered before.

- The cell is bounded by obstacles, so it is not possible to finish it with a bounding line. In this case the robot returns to the last covered area.

To deal with the last two situations the robot needs to maintain a complex map. However if the robot leaves marks along its path it becomes easy to recognise if an area was already covered or not. When the robot senses the presence of previous marks, it stops covering the current cell considering that this part was already covered and thus the whole area beyond the sensed mark was covered too (because the covered area is guaranteed to be solid).

\section{Sensing chemical marks}

In order to detect a cleaned area, the robot must be able to measure very small traces of the cleaning material near the ground. For that purpose, a good spatial resolution of the distribution of the target gas should be achieved. The following main issues are critical for the sensing process:

- Time response of the sensing device: a longer time constant of the sensing device in relation to the cleaning substance leads to a slower detection of the cleaned area.

- Velocity of the robot: the increase of robot's velocity reduces the spatial resolution.

- Evaporation rate: if the evaporation rate of the cleaning material is too fast it will not be possible to detect it after 


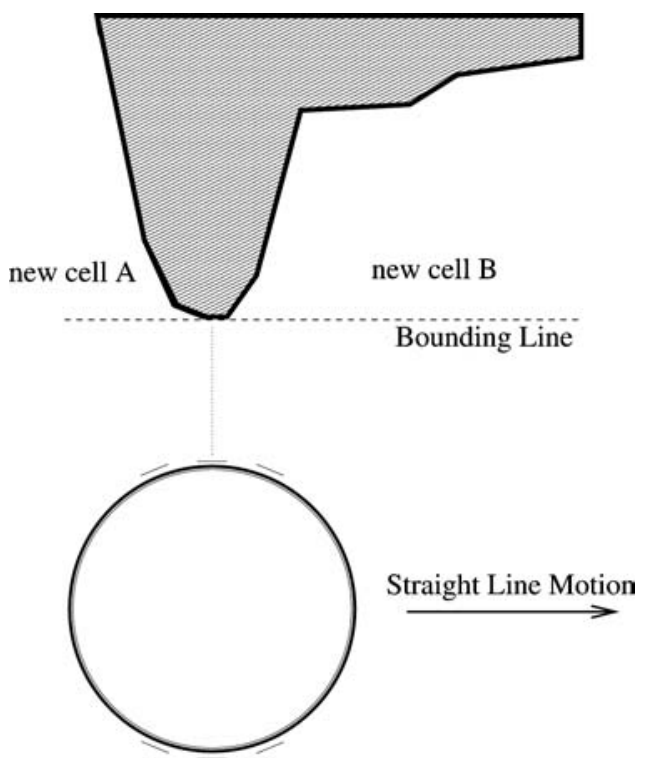

Fig. 4 Scheme of a robot equipped with range sensing devices, and the detection of a bounding point that will define a bounding line separating two new cells

some time. However if it doesn't evaporate enough, the vapour concentration will be too small and the chemical mark will not be detected.

So the task must be performed in a balance point in which these factors fit each other. The evaporation rate restricts the minimum velocity of cleaning because the robot must be able to detect the vapour of the chemical mark before it vanishes. The time constant of the gas sensors, limits the maximum velocity of the robot which must be slow enough to allow the accurate perception of a cleaned area boundary. On the other hand the evaporation rate must be high enough so it can be detected by currently available sensors. The response time of the sensory system is due to the sensor and sampling system response time, added to a delay caused by the rate of gas dispersion.

\subsection{Trace detection}

The local concentration of the odour field is measured with a metal oxide-based electronic nose (see Fig. 6). The output of this system already provides an estimation of the target odour concentration (Marques et al., 2003)., As a first approach, a threshold of the measured value was employed for chemical trace detection. However, experiments have shown large fluctuations of the concentration measured near the boundaries of cleaned areas, giving rise to large instabilities in the detection process. After a deeper analysis of the problem, it was decided to filter the electronic nose output with two sequential low-pass filters tuned with different dynamics-a slower one to capture the odour field average and a faster

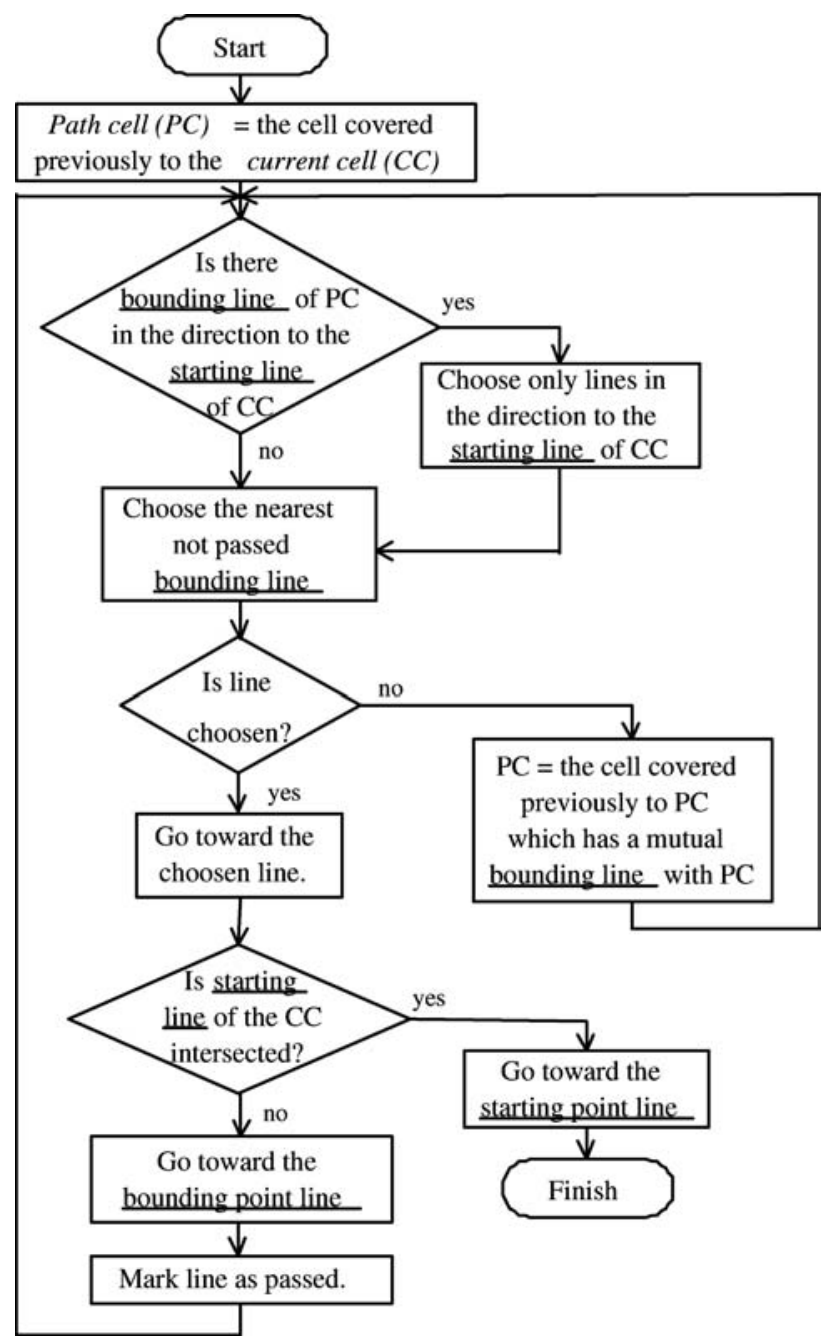

Fig. 5 Path to the starting point of the current cell (for example see Fig. 10)

one to capture changes in concentration that might be representative of a cleaned boundary.

The odour field is modelled as a linear stochastic process $x$ corrupted by zero mean Gaussian white noise $w$ with variance $\sigma_{w}^{2}$. The measures $y$ taken with the electronic nose are also assumed to be corrupted by Gaussian white noise $n$ with variance $\sigma_{n}^{2}$. Basically, $\sigma_{w}$ represents the statistical fluctuation of the odour field and $\sigma_{n}$ represents the error of the electronic nose. The filtered measures are obtained by means of the following general equation:

$\hat{x}_{k}=a \hat{x}_{k-1}+(1-a) y_{k}$

where $0<a<1$ is a filtering constant that establishes the bandwidth of the filter, the higher the value of $a$ the slower the filter will be (Oppenheim and Schafer, 1989).

Figure 7 shows a sample signal obtained while the nose entered inside a cleaned area. The measured concentration was filtered by two filters with different bandwidths. It can 
Fig. 6 Electronic nose used to estimate the odour field concentration near the ground. A vacuum pump is used to draw in air to the nose's sampling chamber

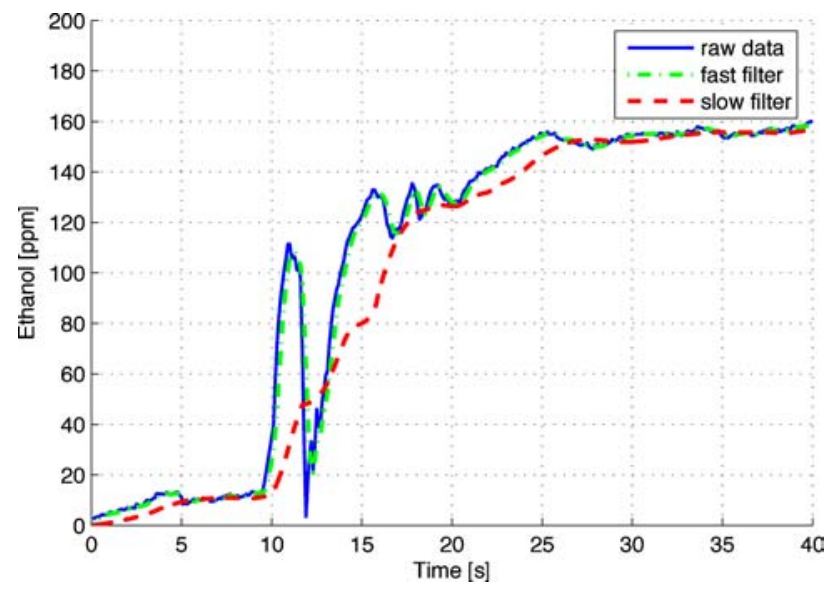

Fig. 7 Odour concentration measured along the robot trajectory filtered with two different bandwidths. It is possible to notice a large instability when the robot entered in the cleaned area. This instability might have been due to changes in the airflow direction

be noticed that the slow filter provides an average level of the signal while the fast filter tracks more closely the signal. The start of the trace is detected when the difference between the fast filter and the slow filter exceeds a threshold. The end of the trace is detected if the difference between the slow filter and the fast filter exceeds the same threshold. This threshold was chosen experimentally and is equal to $25 \mathrm{ppm}$.

There are two sensors situated on the robot. Each of the sensor signals is processed using the algorithm described above giving the output value $v_{i}=1$ if the trace is sensed and $v_{i}=0$ if there is no trace. Combining these two values, the following conclusions about the current position of the robot are made:

- $v_{1}=1$ and $v_{2}=1 \Rightarrow$ this place is cleaned

- $v_{1}=0$ and $v_{2}=0 \Rightarrow$ this place is not cleaned

$-v_{1} \neq v_{2} \Rightarrow$ this place is not cleaned but it was already partially visited

\subsection{Compensation of false detections}

In an ideal case the chemical trace would be sensed only in the places where a robot has leaved a chemical mark. But in real conditions there is always some air convection which disturbs the regular shape of the marked area. This might trigger a trace false detection in places not cleaned yet due to the chemicals transported from the areas already covered. For example, during the work presented in Larionova et al. (2003) a relatively constant airflow at the ground level was observed. This effect, caused by the convection generated by thermal gradients inside our lab, is likely to be found in any indoor environment, since sources of heat are common everywhere (e.g., persons, computers, windows, etc). In order to deal with this problem, the existence of small airflows causing a false detection of a cleaned boundary is considered in the estimation process. The probability of this false detection can be expressed as $P(v=1 \mid$ nomark $)$ probability to detect a trace if there was no mark leaved on the ground.

Another assumption can be derived from the analysis of both electronic noses output: one can be sure that the place is not cleaned if both systems do not sense the presence of a chemical mark, however it is not certain that the place is 
cleaned even if both systems sense that mark due to the previously described disturbances caused by the airflow. Thus two more probabilities are considered:

$P(v=1)$-probability that a mark is sensed, $P$ (nomark) - probability that the place is not cleaned.

These probabilities are assigned as follow:

- The place is cleaned $\Rightarrow P(v=1) \geq 0.9, P($ nomark $) \leq$ 0.5

- The place is not cleaned $\Rightarrow P(v=1)=P(v=$ $1 \mid$ nomark $), P($ nomark $) \geq 0.9$

The equation for $P($ nomark $\mid v=1)$ can be obtained using Bayes' formula. Remembering that $P(\operatorname{mar} k \mid v=1)=$ $1-P($ nomark $\mid v=1)$ the final probability of a mark being present is:

$P(\operatorname{mark} \mid v=1)=1-\frac{P(\text { nomark }) P(v=1 \mid \text { nomark })}{P(v=1)}$

If $P(\operatorname{mark} \mid v=1)>0.5$ then the place is cleaned and not cleaned otherwise. This equation provides a way to reject false detections if the probability $P(v=1 \mid$ nomark $)$ is high. However an estimation of $P(v=1 \mid$ nomark $)$ is not an obvious task because the influence of the airflow is a complex multiparameter process. In this work there is no attempt to measure the small airflows usually present indoors and perform its exact modelling. ${ }^{1}$ Instead, a simple model is proposed:

- Four directions of the airflow relatively to the sweeping direction of the current cell are considered: starting line, front, left and right. A probability of the airflow having one of those directions is considered: $P_{A F}$ (start), $P_{A F}$ (front), $P_{A F}(l e f t), P_{A F}(r i g h t)$.

- A probability that a mark was already placed in each of the possible airflow directions is considered: $P_{\text {Mark }}\left(\right.$ start), $P_{\text {Mark }}($ front $), P_{\text {Mark }}($ left $), P_{\text {Mark }}$ (right).

- The presence of a mark and the airflow are independent events for each direction, so, the whole probability to sense a mark due to airflow is:

$$
\begin{aligned}
P(v=1 \mid \text { nomark })=P_{A F} & (\text { start }) \cdot P_{\text {Mark }}(\text { start }) \\
& +P_{A F}(\text { front }) \cdot P_{\text {Mark }}(\text { front }) \\
& +P_{A F}(\text { left }) \cdot P_{\text {Mark }}(\text { left }) \\
& +P_{A F}(\text { right }) \cdot P_{\text {Mark }}(\text { right })
\end{aligned}
$$

\footnotetext{
${ }^{1}$ in fact it is very difficult to measure airflows of less than $10 \mathrm{~cm} / \mathrm{s}$ with common anemometers.
}

The airflow probabilities $P_{A F}$ are constantly updated during straight-line motions using two kind of events which are easily determined:

There is no airflow: occurs if the sensors conclude that there is no mark. In this case the $P_{A F}$ is decreased if the corresponding $P_{\text {Mark }}>0.5$

$$
P_{\text {AFnew }}=P_{A F} \cdot\left(1-0.01 \cdot P_{\text {Mark }}\right)
$$

There is airflow: occurs if the mark was sensed during some (short) period of time. This means that when a mark is unstable, it is probably caused by airflow. In this case the $P_{A F}$ is increased:

$$
P_{\text {AFnew }}=P_{A F} \cdot\left(1+0.25 \cdot P_{\text {Mark }}\right)
$$

The values of $P_{A F}$ are adjusted in order to guarantee that their sum is less or equal to 1 . The probabilities $P_{\text {Mark }}$ are determined according to the current position of the robot in the cell. $P_{\text {Mark }}($ start $)=0.9$ in all cases because the area between the robot position and the starting line is already covered. If the robot moves from left to the right then $P_{\text {Mark }}($ left $)=0.9$ and $P_{\text {Mark }}($ right $)=0.5$ (not known), and vice versa in the opposite case. $P_{\text {Mark }}($ front $)=0.5$ because there is no information if the area in front is already covered or not. If there is an obstacle closer than 1 meter to the robot then the appropriate $P_{\text {Mark }}$ is decreased in order to become zero when the distance to obstacle is also null (extreme case).

\section{Simulation}

A Nomad Super Scout II robot model was used in simulation and all simulation tests have been performed using Nomadic simulation software (Nomadic, 1999). The simulation was performed under the following assumptions:

- Gas sensor: there are two ideal gas sensors, positioned at the edge of the robot in both sides at $30^{\circ}$ from the front.

- The field caused by the cleaning chemicals is transformed in a binary grid in which values can be either 1 (cleaned) or 0 (not cleaned).

- The map used to perform the simulation is external (i.e., the robot has no knowledge about it).

The simulation environment provides the response of robot's range sensors during its path from simulated sonars. The simulated robot is controlled by the program that implements the proposed algorithm, and also simulates the olfaction field. The simulation map is shown on Fig. 11. 


\section{W00000000 9000000000000000000000000}

Fig. 8 Cover of cell 1, stop at the end point and make two new cells (2 and 3)

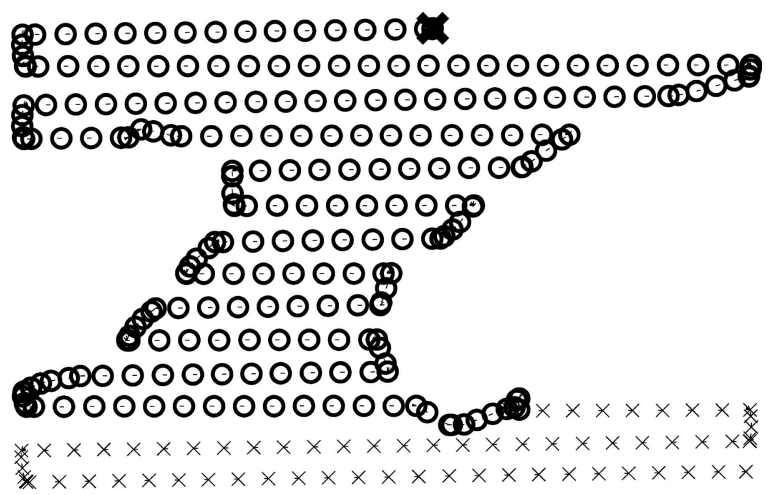

Fig. 9 Move to the start of cell 2 and cover it until next end point, make four new cells $(4,5,6$, and 7$)$

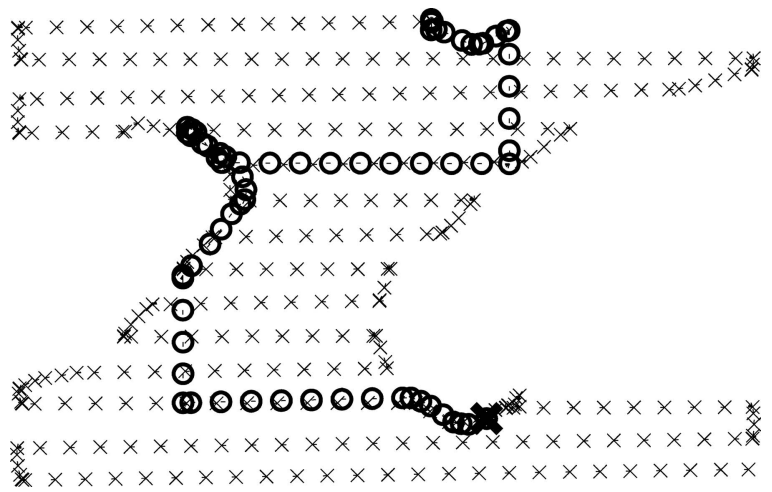

Fig. 10 Path to the starting point of cell 3 (see Fig. 5)

Figures 8 to 11 show the results of covering the simulated area.

\section{Practical implementation}

\subsection{Robots and noses}

The proposed algorithm was tested in a Nomad Super Scout II mobile robot equipped with a cleaning device and two electronic noses (see Fig. 12). The cleaning device (A) consists in a sponge embedded with a cleaning solution containing ethanol. The cleaning solution is stored in a bottle (B) placed in the top of the robot. The liquid flow, pumped to the sponge by gravity, is adjusted by means of a manual valve. The electronic noses (D) are located in front of the robot and draw air at about $5 \mathrm{~cm}$ height from the floor by means of miniature vacuum pumps (Sensidyne AAA series micro-pump) located in the top of the robot (C).

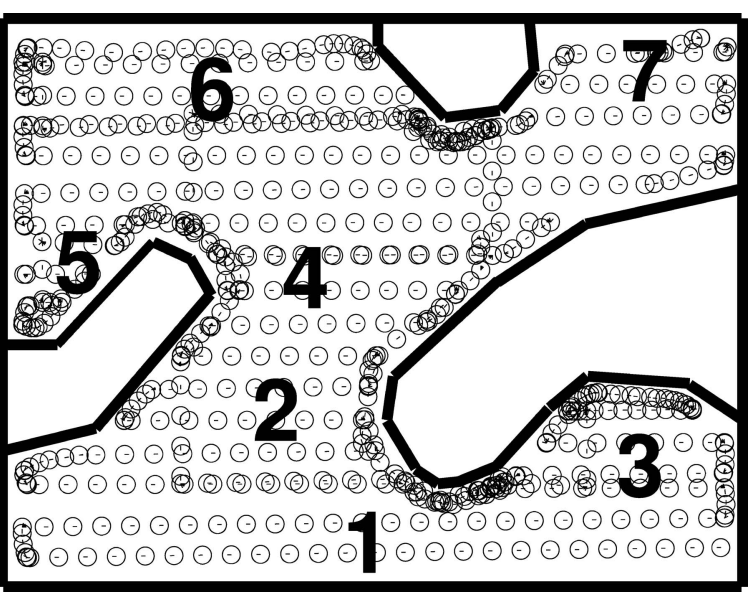

Fig. 11 Complete coverage attained. Map of the environment and numbers of cells

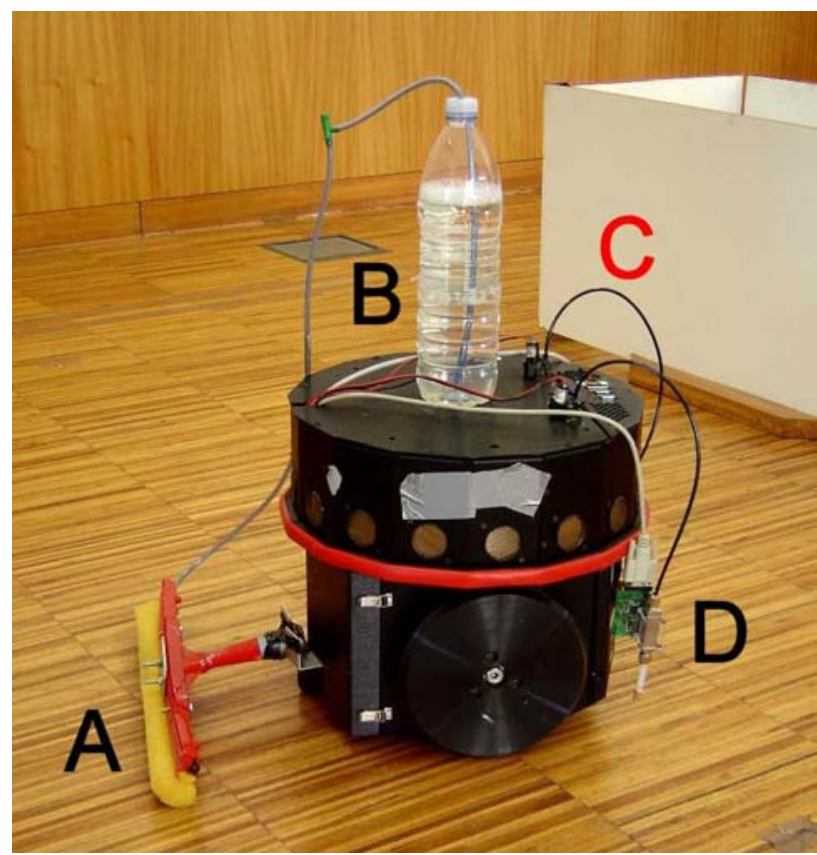

Fig. 12 Mobile robot equipped with cleaning device and chemical sensing nostrils

The electronic nose is based in an array of 4 tin oxide gas sensors (Figaro series TGS26xx). This system allows operating the sensing array in multiple temperatures, increasing the sensing space, and allows processing the gas sensors' transient response in order to increase the system response time (Almeida et al., 2003; Marques et al., 2003).

In a previous work (Larionova et al., 2003), the smell of the ground floor was acquired using an exposed nostril and a set of three fans arranged in order to generate an airflow current from the ground up to the nostril. This arrangement, similar in concept to the one proposed by Russell (1995), generated a high degree of turbulence, worsening the estimation of cleaned area boundaries. The current version, 
using vacuum pumps to draw in air from the ground surface to an electronic nose, does not disturb the odour field, allowing a much more precise identification of the cleaned areas boundaries.

\subsection{Range sensors}

For this work acoustic sonars of the Nomad Super Scout II robot were used for sensing the range between the robot and the surrounding obstacles. During the experimental tests performed inside the laboratory, these sonars were not able to provide adequate information about range because of crosstalk and non-perpendicularity between the sonar beams and the obstacle's surfaces. To overcome these problems an occupancy grid map was used (Elfes, 1989). During the straight motion of the robot two grid maps are created: one using sonars from the left side of the robot, and another using sonars from the right. At the end of a straight motion these maps are analysed to determine the points where bounding slicing lines must be created.

\subsection{Experimental results}

The tests were performed under the following controlled conditions to limit the airflow and turbulence near the ground.

- A test area with dimensions $410 \times 410 \mathrm{~cm}^{2}$ with an obstacle at the centre (see Fig. 13).

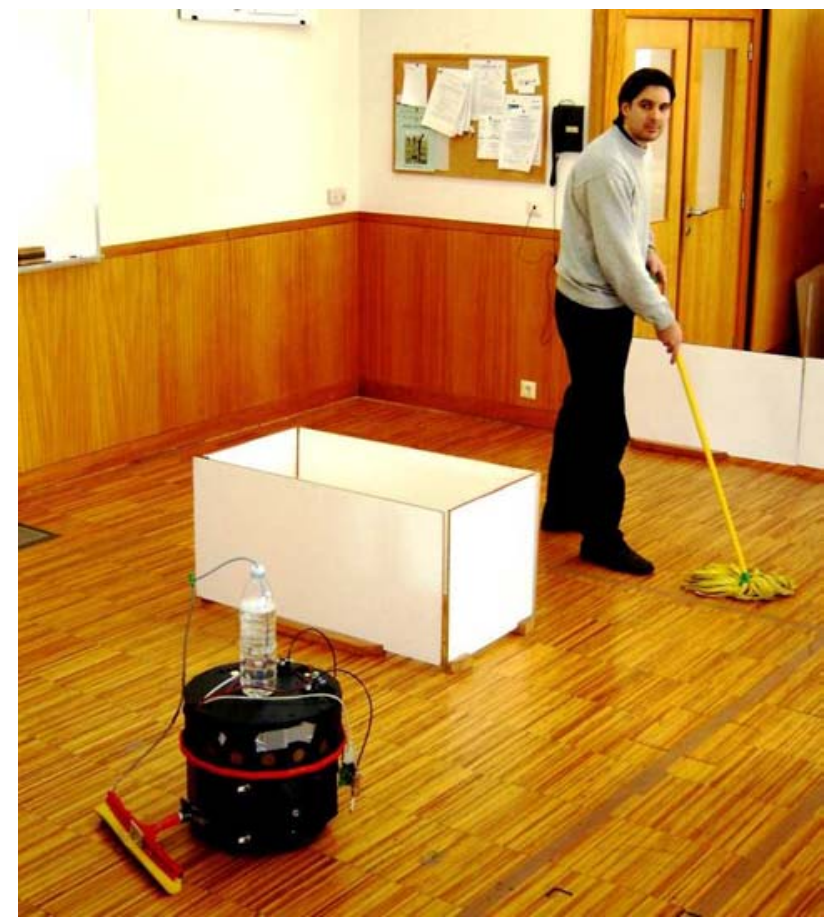

Fig. 13 Real test: a human cleaner sweeps a region of the map. This area must not be covered by the cleaning robot

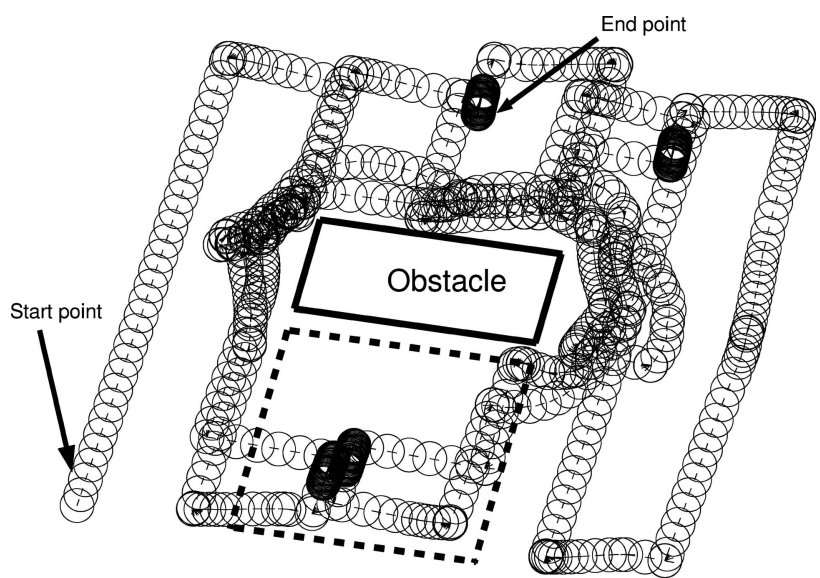

Fig. 14 Coverage test: the robot successfully covers the area which has not yet been cleaned. The dashed area, which was formerly clean, was not completely covered

- To create a detectable cleaning solution, a weak alcohol solution (10\%) was used.

- A cleaning tool was attached to the back of the robot allowing the release of the chemical mixture along the robot trajectory.

- The release rate to the sponge was adjusted with a manual valve to approximately $15 \mathrm{ml} / \mathrm{min}$.

- The robot was moved at low velocities ( $v_{\text {robot }} \leq 2 \mathrm{~cm} / \mathrm{s}$ ).

- The electronic noses provided estimations of the target odour concentration every 0.5 seconds.

To illustrate the capability of cooperation, a human cleaner has previously swept a partial area of the map. After this, the robot starts cleaning other parts of the remaining area. The robot cleaned all the uncleaned cells as established by the algorithm, and when it entered the already cleaned area it detected the chemical trace and stopped cleaning the current cell, passing to the next one. The resultant trajectory is shown in Fig. 14.

\section{Conclusions and future work}

The proposed algorithm based in exact cellular decomposition and olfaction information has proved it effectiveness both by simulation and by experimental tests. Experimental coverage tests with unknown areas demonstrated the validity of the proposed solution.

The odour field is accurately acquired with a noninterfering tin oxide sensor based electronic nose coupled to a miniature vacuum pump. A method to improve the estimation of the cleaned areas boundaries taking into account the effects of small airflow currents, always present in indoor environments, was proposed.

In the future the algorithm will be adapted to work in dynamic environments, and will be tested in a real 
multi-robot environment. The robustness of the current solution will be enhanced using multiple sensing modalities to sense the areas already cleaned (e.g., chemical sensors plus optical detectors to measure the ground reflection and detection of dust near the floor) and adapting sensitive airflow sensors around the robot to estimate the airflow field.

Acknowledgments This research was supported by the FCT (Portuguese Foundation for Science and Technology) under the project RoboNose

\section{References}

Acar, E. and Choset, H. 2000. Critical point sensing in unknown environments. In Proc. IEEE Int. Conf. on Robotics and Automation, pp. 3803-3810.

Almeida, N., Marques, L., and de Almeida, A. 2003. Fast identification of gas mixtures through the processing of transient responses of an electronic nose. In Proc. of EuroSensors.

Choset, H. 2001. Coverage for robotics-A survey of recent results. Annals of Mathematics and Artificial Intelligence, 31:113-126.
Choset, H. and Pignon, P. 1997. Coverage path planning: The boustrophedon decomposition. In Int. Conf. on Field and Service Robotics.

Elfes, A. 1989. Using occupancy grids for mobile robot perception and navigation. Computer, 22:46-57 (Special Issue on Autonomous Intelligent Machines).

Gabriely, Y. and Rimon, E. 2002. Spiral-STC: An on-line coverage algorithm of grid environments by a mobile robot. In Proc. IEEE Int. Conf. on Robotics and Automation, pp. 954-960.

Hert, S., Tiwari, S., and Lumelsky, V. 1996. A Terrain-covering algorithm for an AUV. Autonomous Robots, 3:91-119 (Special Issue on Autonomous Underwater Robots).

Larionova, S., Almeida, N., Marques, L., and de Almeida, A. 2003. Olfactory coordinated area coverage. In Proc. IEEE Int. Conf. on Advanced Robotics, pp. 501-506.

Mann, G. and Katz, G. 1999. Chemical trail guidance for floor cleaning machines. In Int. Conf. on Field and Service Robotics.

Marques, L., Almeida, N., and de Almeida, A. 2003. Olfactory sensory system for odour-plume tracking and localization. In IEEE Int. Conf. on Sensors, Toronto, Canada.

Nomadic. 1999. Nomad Scout User's Manual, Nomadic Technologies Inc.

Oppenheim, A. and Schafer, R. 1989. Discrete-Time Signal Processing, Prentice-Hall.

Russell, R. 1995. Laying and sensing odor markings as a strategy for assisting mobile robot navigation tasks. 2(3):3-9.

Svennebring, J. and Koenig, S. 2003. Trail-laying robots for robust terrain coverage. In Proc. IEEE Int. Conf. on Robotics and Automation. 